Article

\title{
Data Mining and NIR Spectroscopy in Viticulture: Applications for Plant Phenotyping under Field Conditions ${ }^{\dagger}$
}

\author{
Salvador Gutiérrez, Javier Tardaguila, Juan Fernández-Novales and Maria P. Diago * \\ Instituto de Ciencias de la Vid y del Vino (University of La Rioja, CSIC, Gobierno de La Rioja) Ctra. De Burgos \\ Km, 6, 26007 Logroño, Spain; salvador.gutierrez@unirioja.es (S.G.); javier.tardaguila@unirioja.es (J.T.); \\ juan.fernandezn@unirioja.es (J.F.-N.) \\ * Correspondence: mpaz.diago.santamaria@gmail.com; Tel.: +34-94-189-4980 (ext.410065); Fax: +34-94-189-9728 \\ + This paper is an extended version of our paper published in Proceedings of the 2nd International Electronic \\ Conference on Sensors and Applications, online, 15-30 November 2015.
}

Academic Editor: Dirk Lehmhus

Received: 2 December 2015; Accepted: 4 February 2016; Published: 16 February 2016

\begin{abstract}
Plant phenotyping is a very important topic in agriculture. In this context, data mining strategies may be applied to agricultural data retrieved with new non-invasive devices, with the aim of yielding useful, reliable and objective information. This work presents some applications of machine learning algorithms along with in-field acquired NIR spectral data for plant phenotyping in viticulture, specifically for grapevine variety discrimination and assessment of plant water status. Support vector machine (SVM), rotation forests and M5 trees models were built using NIR spectra acquired in the field directly on the adaxial side of grapevine leaves, with a non-invasive portable spectrophotometer working in the spectral range between 1600 and $2400 \mathrm{~nm}$. The $v$-SVM algorithm was used for the training of a model for varietal classification. The classifiers' performance for the 10 varieties reached, for cross- and external validations, the $88.7 \%$ and $92.5 \%$ marks, respectively. For water stress assessment, the models developed using the absorbance spectra of six varieties yielded the same determination coefficient for both cross- and external validations $\left(R^{2}=0.84\right.$; RMSEs of 0.164 and $0.165 \mathrm{MPa}$, respectively). Furthermore, a variety-specific model trained only with samples of Tempranillo from two different vintages yielded $\mathrm{R}^{2}=0.76$ and RMSE of $0.16 \mathrm{MPa}$ for cross-validation and $\mathrm{R}^{2}=0.79$, RMSE of $0.17 \mathrm{MPa}$ for external validation. These results show the power of the combined use of data mining and non-invasive NIR sensing for in-field grapevine phenotyping and their usefulness for the wine industry and precision viticulture implementations.
\end{abstract}

Keywords: variety classification; plant water status; non-destructive; SVM; rotation forest; regression tree; stem water potential

\section{Introduction}

In the context of the current worldwide industrial demand of quality and efficiency in crop and food production, the importance of phenotyping arises every day. Plant phenotyping refers to a quantitative description of the plant's physiological, biochemical and morphological properties, among others [1]. It consists of the identification of effects on the phenotype as a result of genotype differences and the environmental conditions to which a plant has been exposed [2]. The development of new usable technologies and its direct availability have driven the latest plant phenotyping approaches that have emerged and have already been applied in several environments [3]. These technologies have enabled the performance of phenotyping tasks with reduced time and monetary costs (much sought after by the industrial actors) and remain under the focus of researchers from different currents 
of investigation, trying especially to provide realistic, applicable and suitable solutions. Proximal sensing approaches, such as spectroscopy sensors or hyperspectral imaging, have arisen in the last few years as fast, non-destructive resources for the gathering of crop spectral information that could characterize concrete phenotyping traits, providing the in-field methods with a high relevance due to their desirable capability of providing in situ results.

Viticulture has benefited from these results of recent research that have developed methods and procedures for several vine- and wine-related problems using near-infrared (NIR) spectroscopy. NIR spectroscopy is a potent technology widely used in several agricultural areas due to its non-destructive nature and multi-parametric capabilities [4]. Spectroscopic sensors have been proven to be fast for the real-time assessment of several grapevine-related traits, such as the grape composition [5], the grapevine petiole nutrient concentration assessment [6] or the identification of grape berry sunburn symptoms [7]. Therefore, the possibility of the use of NIR technology for grapevine phenotyping arises as an attractive and promising tool for precision viticulture, especially when taking into account the fact that this technique is able to characterize more than one parameter using the same spectral measurement.

NIR devices are able to acquire large amounts of spectral data, making it necessary to manage them in efficient and automatic ways. Data mining has become one of the most valuable research fields in the latest few years due to its knowledge discovery power, direct applicability in several areas and, especially, its proven effectiveness in those problems where it is applied. Data mining through, among others, machine learning techniques have provided procedures for both descriptive (characterizations of the properties of the data) and predictive (learning and induction of the data for forecasting) tasks $[8,9]$. Some of the most widespread applications of predictive techniques are decision trees [10], decision forests [11] and, particularly, artificial neural networks (ANNs) [12] and support vector machines (SVMs) [13], employed in several research areas, such as medicine [14], business and industry [15] or biology [16]. Support vector machines [13,17] are supervised learning methods used for classification and regression through the nonlinear mapping of the input data. SVMs transform the original dataset into a higher dimension using a kernel function and find an optimal separating hyperplane, the best one that maximally separates the samples. Rotation forests [18] are machine learning ensemble methods that make use of several classification trees (hence the name) to build a meta-classifier. A rotation forest can be used both for classification or regression, depending on the kind of tree-based algorithm used. A robust regression tree is the M5 learner [19], which, although not as familiar as other estimation methods in spectroscopy, like partial least squares (PLS) [20], has demonstrated robustness and efficiency in other applications, such as pan evaporation prediction [21], low-flow forecasting modeling [22] or the water level-discharge relationship [23].

Two important grapevine phenotyping topics are varietal discrimination and water status assessment, tasks addressed in the literature and where spectroscopy especially has played a significant part in the last few years. Current varietal discrimination methods have some lack of aspects that are relevant for an industrial point of view, e.g., their need for a highly trained expert or their destructive nature [24]. Water status assessment especially suffers from this last issue, as well as its time and labor-consuming nature, along with the lower representative capacity (limited number of samples measured) derived from it [25]. Grapevine varietal discrimination using spectroscopic data has been recently attempted by hyperspectral imaging under laboratory conditions [24]. Both in-lab or in-field water status assessment via spectroscopy have also been aimed at, attending to several plant water condition indicators, such as stem water potential [26,27], leaf water potential $[26,28,29]$ or leaf stomatal conductance [26]. It is worth highlighting that each and every one of the mentioned studies has one common factor: the use of partial PLS as the model training algorithm. PLS is a widespread statistical technique commonly used in spectroscopy for the regression of chemometric parameters. Qualitative prediction (e.g., discrimination among discrete classes) can also be achieved using PLS (as in [30], where a binary classification is translated into a regression of two natural numbers) or via a purest discrete classification method, like partial least squares discriminant analysis (PLS-DA) [31]. Still, 
discrimination models built with PLS-based approaches have not yielded remarkable results when taking into account a considerably large amount of classes. Hence, the attractive attempt to apply less often used data mining techniques for the modeling of NIR spectra, thus making it possible to carry fast, in-field solutions for these two grapevine phenotyping approaches into commercial and industrial demands.

The goal of this study was to evaluate the combined use of different data mining techniques along with a non-destructive NIR portable sensor for the in-field grapevine phenotyping of two concrete traits: the variety classification and the estimation of the plant water status.

\section{Experimental Section}

\subsection{Experimental Layout, Acquisition of NIR Spectra and Reference Measurements}

Two experiments regarding grapevine varietal classification and plant water status were conducted.

Both experiments were carried out during late August and early September 2012 in a vineyard located in Vergalijo, Navarra, Spain (latitude $42^{\circ} 27^{\prime} 45.96^{\prime \prime} \mathrm{N}$, longitude $1^{\circ} 48^{\prime} 13.42^{\prime \prime} \mathrm{W}$, altitude $325 \mathrm{~m}$ ). Vines of different varieties were planted in 2009 and trained to a vertical shoot-positioned trellis system at $2 \times 1 \mathrm{~m}$ inter- and intra-row distances, with a north-south row orientation.

For both experiments, spectra acquisition was performed in the field with an integrated portable NIR spectral analyzer (microPHAZIR ${ }^{\mathrm{TM}}$, Thermo Fisher Scientific Inc., Waltham, MA, USA) operating in the range of 1600 to $2400 \mathrm{~nm}$ with a step of $8.7 \mathrm{~nm}$ (a total of 100 data points per spectrum). All spectra were returned by the device in absorbance mode and in this form were used for analysis.

For the grapevine varietal classification, 10 varieties were used: Cabernet Sauvignon, Caladoc, Carmenere, White Grenache, Pedro Ximenez, Pinot Noir, Tempranillo, Treixadura, Viognier and Viura. For each variety, 10 vines and two adult leaves per vine were selected from the mid-upper part of the shoot (Nodes 6 to 12) for the measurement of its spectrum on the adaxial side, making up a total of 20 leaves per variety. Five spectra per leaf (from different positions of the surface) were taken, and their average was considered the final spectrum of that leaf. Therefore, a total of 200 leaves (10 varieties, 20 leaves per variety) were used, and the name of the corresponding variety was linked to each measurement for the training of the varietal classification model.

For the assessment of the grapevine water status, measurements were carried out in six varieties during two days: Godello, Grenache, Pedro Ximenez (29 August 2012; vapor pressure deficit: $0.87 \mathrm{kPa}$; average temperature: $21.7{ }^{\circ} \mathrm{C}$; average relative humidity: $68 \%$ ), Carmenere, Marselan and Tempranillo (5 September 2012; vapor pressure deficit: $0.89 \mathrm{kPa}$; average temperature: $19.8{ }^{\circ} \mathrm{C}$; average relative humidity: $62 \%$ ). As in the varietal discrimination experiment, 10 vines and two adult leaves per vine were selected from the mid-upper part of the shoot (Nodes 6 to 12) for each variety. A total of 120 leaves ( 6 varieties, 20 leaves per variety) were measured, thus for the training of the water status assessment model. Spectra acquisition was done on the adaxial side of the leaves. Afterwards, the midday stem water potential $\psi_{\text {stem }}$ (14:00, solar noon) of each leaf was measured as the reference method of water stress [32]. Stem water potential was determined using a Scholander pressure bomb (Model 600, PMS Instruments Co., New York, NY, USA). The selected leaves were driven into dark adaptation by covering them with aluminum foil bags one hour before the $\psi_{\text {stem }}$ measurement.

In order to test the robustness of the algorithms, a second model for water status assessment was developed involving samples of a given variety (to analyze the $\psi_{\text {stem }}$ prediction capability within one variety) acquired at different seasons and vineyards (to test the prediction capability with samples at different phenological stages). Thirty-six leaf spectral measurements of Tempranillo were acquired in 12 August 2015 in a vineyard located in Tudelilla, La Rioja (42 ${ }^{\circ} 18^{\prime} 17.9208^{\prime \prime},-2^{\circ} 7^{\prime} 15.8376^{\prime \prime}$; vapor pressure deficit: $1.74 \mathrm{kPa}$; average temperature: $26.7^{\circ} \mathrm{C}$; average relative humidity: $53 \%$ ) using the same procedure as in 2012. A new variety-specific dataset for Tempranillo was built up merging these 36 samples and the 20 samples of Tempranillo taken in 2012, making up a total of 56 samples. 
For both variety discrimination and water status assessment, the optical window of the NIR device was fully covered and vinyl gloves were used when taking the measurements in order to avoid contamination from external light and pollutants.

\subsection{Spectra Pre-Processing and Data Mining Algorithms}

\subsubsection{Pre-Processing}

An outlier detection analysis was performed before any other spectral treatment. It consisted of the following procedure: at measurement time, the acquired spectrum was compared by the sensor with a previously taken grapevine leaf's spectrum signature and labeled according to whether the signal was from a leaf or not. All spectra that did not belong to a grapevine leaf were treated as outliers and thus removed.

Scatter correction and spectral derivative were applied to the raw spectra, in order to diminish the physical variability between samples because of scatter and to remove both additive and multiplicative effects in the spectra, respectively [33]. Standard normal variate (SNV) followed by de-trending [34,35] was used as a scatter correction method. Afterwards, a Savitzky-Golay smoothing and derivative process [36] was applied with a window size of five and a second-degree derivative.

\subsubsection{Data Mining Algorithms}

Due to the different nature of the phenotyping features addressed in this work, distinct data mining algorithms for classification and regression were applied for grapevine varietal discrimination and water status assessment, respectively.

\section{Varietal Discrimination}

For grapevine varietal discrimination, SVMs were used. The $v$-SVM algorithm [37] was used in this study, implemented in LIBSVM [38], the post-processed spectra data points, linked with its variety label (the class), being the input of the algorithm. A second-degree polynomial kernel and a value of 0.1 were set as the parameters of the algorithm.

The classifiers evaluation was performed attending not only to the confusion matrix, but also to a deeper analysis involving the true and false positive rates, precision values and receiver operating characteristic (ROC) curves' area (area under the curve, AUC). The true positive rate refers to the proportion of samples that were discriminated as a specific class among all samples, which truly corresponded to that class (it is similar to the correctly classified percentage divided by 100). The false positive rate is the proportion of samples that were classified as a specific class, but belonged to a different one, among all examples that were not of that class. The precision represents the proportion of the samples that were correctly classified in their class among all those that were classified as that given class. The AUC is the measure of the area that lies under the ROC curve. An ROC curve is a graphic representation of true positive versus false positive rates by varying a given threshold. ROC curves' AUC is a common metric for the evaluation of a classifier. A perfect classifier would achieve an AUC of 1 , while a system that classifies instances in a random way would obtain a 0.5 value.

Water Status Assessment

To address the grapevine water status assessment, regression with rotation forest and M5 trees was applied. The spectral data points were used as the input of the algorithm and each sample's $\psi_{\text {stem }}$ measurement as the value to predict. Weka software, Version 3.6, [39] was used for the development of the regression models.

\section{Algorithm Validation}

For the assessment of the algorithms' results, calibration, cross-validation and external testing were carried out. For each one of the experiments, its dataset was divided into two subsets, training 
and test, comprising $80 \%$ and $20 \%$ of the original samples, respectively. The test set was never used for the training of any of the models. In the calibration assessment, the models were developed using the training set and validated with the same one. In the cross-validation, a $k$-fold method was performed upon the training dataset with a $k$ value of 5 (in order to maintain the 80:20 ratio, obtaining five executions where, in each one of them, the model is trained with $80 \%$ of the samples and evaluated testing the remaining 20\%). Finally, the prediction results were obtained via an external validation, training and testing the models with the training and test datasets, respectively. The test set was obtained in a stratified way (e.g., the same number of samples for each grapevine variety was selected). For the varietal classification, 160 and 40 samples were assigned to the training and test datasets, respectively. For the water status assessment, the multi-variety models' datasets contained 96 and 24 instances, respectively, while the Tempranillo-specific models' datasets involved 45 samples for the training subset and 11 samples for the test subset.

Figure 1 shows a diagram of the datasets and the different calibration and validation processes used in both experiments.

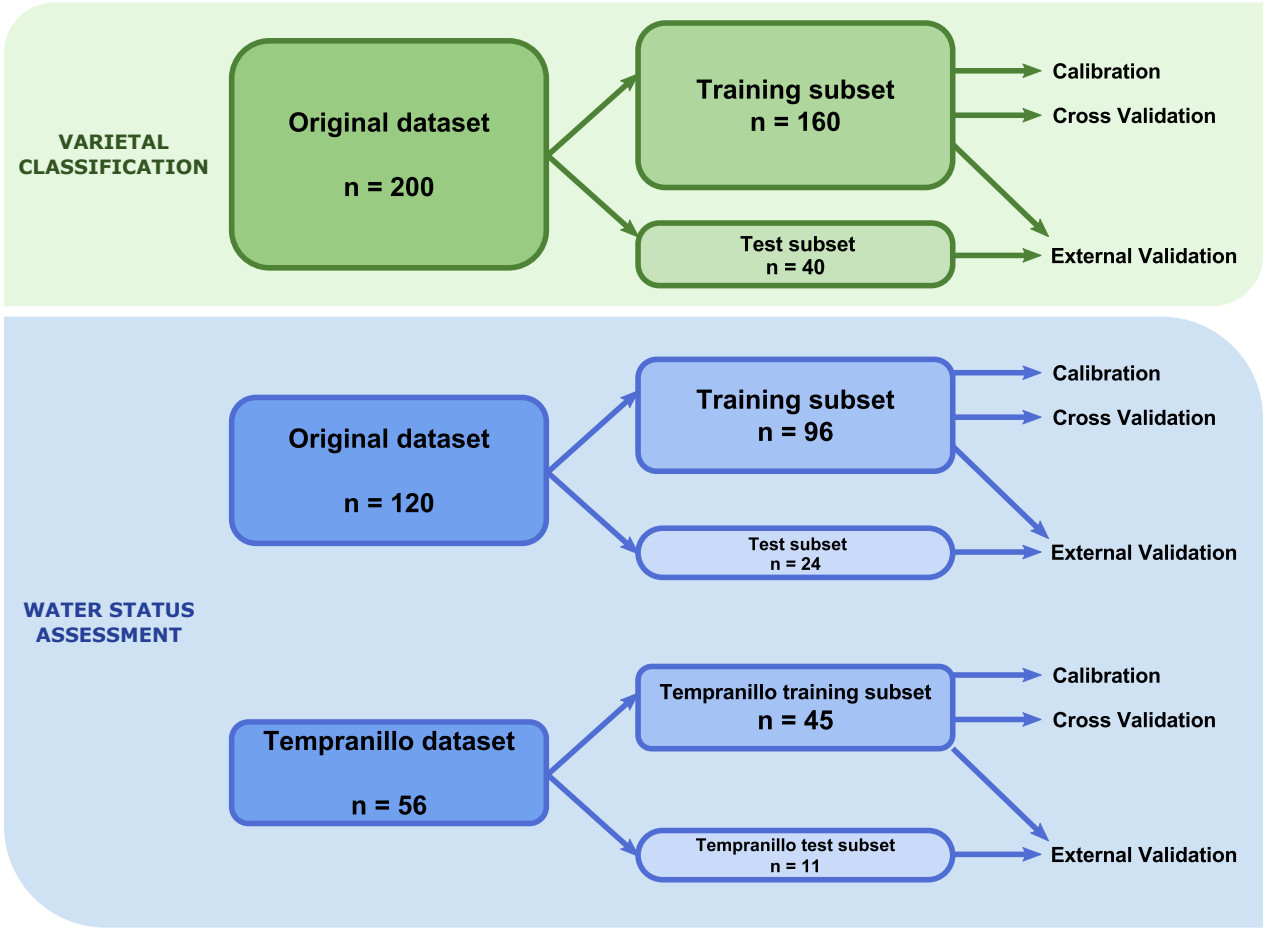

Figure 1. Diagram of the datasets used in both experiments and the different calibration and validation processes.

\section{Results}

\subsection{Grapevine Varietal Discrimination}

The spectral outlier analysis performed before the development of the models resulted in the removal of one sample of White Grenache due to spectral mismeasurement. One hundred percent of correctly classified samples were obtained in the calibration of the SVM classifier trained with the training dataset, reaching perfect scores in the confusion matrix (data not shown). Table 1 presents the confusion matrix from the 5-fold cross-validation of the SVM classifier trained with the training dataset. One hundred forty-one samples out of 159 were successfully discriminated (88.7\%). The confusion matrix shows that the Cabernet Sauvignon variety obtained a perfect score in its discrimination, while all other varieties were above the $80 \%$ mark, excluding the Viognier variety, which obtained a score of $75 \%$ (12 out of 16 correctly discriminated samples). 
Table 1. Confusion matrix of grapevine varietal classification using support vector machines and a 5 -fold cross-validation. The diagonal of the matrix corresponds to the number of samples that were properly classified. The last column displays, for each variety, the correctly classified percentage $(\mathrm{n}=159)$.

\begin{tabular}{ccccccccccccc}
\hline & \multicolumn{10}{c}{ Predicted Variety } \\
\cline { 3 - 14 } & CS & $\mathbf{1 6}$ & CL & CR & WG & PX & PN & TE & TR & VO & VU & \% \\
\cline { 3 - 14 } Actual variety & CL & 0 & $\mathbf{1 5}$ & 0 & 0 & 0 & 0 & 0 & 0 & 0 & 0 & $\mathbf{1 0 0 . 0}$ \\
& CR & 1 & 0 & $\mathbf{1 5}$ & 0 & 0 & 0 & 0 & 0 & 0 & 0 & $\mathbf{9 3 . 8}$ \\
& WG & 0 & 0 & 0 & $\mathbf{1 2}$ & 0 & 0 & 1 & 0 & 2 & 0 & $\mathbf{8 0 . 0}$ \\
& PX & 0 & 1 & 0 & 0 & $\mathbf{1 3}$ & 0 & 0 & 0 & 1 & 1 & $\mathbf{8 1 . 3}$ \\
& PN & 0 & 0 & 0 & 1 & 0 & $\mathbf{1 4}$ & 0 & 0 & 0 & 1 & $\mathbf{8 7 . 5}$ \\
& TE & 0 & 0 & 0 & 0 & 0 & 1 & $\mathbf{1 5}$ & 0 & 0 & 0 & $\mathbf{9 3 . 8}$ \\
& TR & 0 & 0 & 1 & 0 & 0 & 1 & 0 & $\mathbf{1 4}$ & 0 & 0 & $\mathbf{8 7 . 5}$ \\
& VO & 0 & 1 & 0 & 0 & 2 & 0 & 1 & 0 & $\mathbf{1 2}$ & 0 & $\mathbf{7 5 . 0}$ \\
& VU & 0 & 0 & 0 & 0 & 1 & 0 & 0 & 0 & 0 & $\mathbf{1 5}$ & $\mathbf{9 3 . 8}$ \\
\hline
\end{tabular}

CS: Cabernet Sauvignon; CL: Caladoc; CR: Carmenere; WG: White Grenache; PX: Pedro Ximenez; PN: Pinot Noir; TE: Tempranillo; TR: Treixadura; VO: Viognier; VU: Viura.

Table 2. Detailed accuracy by class of the grapevine varietal classification using support vector machines and a 5-fold cross-validation $(n=159)$.

\begin{tabular}{lcccc}
\hline Class & True Positive Rate & False Positive Rate & Precision & AUC \\
\hline Cabernet Sauvignon (CS) & 1.000 & 0.007 & 0.941 & 0.997 \\
Caladoc (CL) & 0.938 & 0.014 & 0.882 & 0.997 \\
Carmenere (CR) & 0.938 & 0.007 & 0.938 & 0.998 \\
White Grenache (WG) & 0.800 & 0.007 & 0.923 & 0.985 \\
Pedro Ximenez (PX) & 0.813 & 0.021 & 0.813 & 0.980 \\
Pinot Noir (PN) & 0.875 & 0.014 & 0.875 & 0.976 \\
Tempranillo (TE) & 0.938 & 0.014 & 0.882 & 0.997 \\
Treixadura (TR) & 0.875 & 0.000 & 1.000 & 0.999 \\
Viognier (VO) & 0.750 & 0.028 & 0.750 & 0.992 \\
Viura (VU) & 0.938 & 0.014 & 0.882 & 0.992 \\
\hline Weighted average & 0.887 & 0.013 & 0.888 & 0.991 \\
\hline
\end{tabular}

AUC: area under the receiver operating characteristic (ROC) curve.

Table 2 shows a detailed accuracy analysis by class for the SVM classifier in the cross-validation process. Similar to the confusion matrix's correctly discriminated percentage (Table 1), one class obtained the highest value for the true positive rate (Cabernet Sauvignon). Nevertheless, due to a sample of the Carmenere class incorrectly assigned to the Cabernet Sauvignon class, the precision value of this variety did not reach the maximum. Additionally, although the Treixadura variety did not obtain a perfect true positive score, the fact that no other instance was classified as that variety (Table 1) led to a perfect precision mark for this variety class. The average AUC yielded by the cross-validation was 0.991 , moving between 0.976 and 0.999 (achieved by the Treixadura variety) for class-specific values.

Table 3 shows the confusion matrix from the external validation of the SVM classifier, where the model was trained using 159 samples and validated with the prediction of 40 external ones. The average correctly discriminated percentage was slightly higher than that of the 5-fold cross-validation, reaching the $92.5 \%$ mark, where 37 out of 40 instances were properly classified. Seven varieties obtained $100 \%$ correctly classified samples, while Pinot Noir, Tempranillo and Viognier had one misclassified sample, dropping their percentage to $75 \%$. 
Table 3. Confusion matrix of grapevine varietal classification using support vector machines and an external validation of 40 samples. The diagonal of the matrix corresponds to the number of samples that were properly classified. The last column displays, for each variety, the correctly classified percentage $(n=40)$.

\begin{tabular}{ccccccccccccc}
\hline & \multicolumn{10}{c}{ Predicted Variety } \\
\cline { 2 - 13 } & & CS & CL & CR & WG & PX & PN & TE & TR & VO & VU & \% \\
\cline { 2 - 13 } Actual variety & $\mathbf{4}$ & 0 & 0 & 0 & 0 & 0 & 0 & 0 & 0 & 0 & $\mathbf{1 0 0 . 0}$ \\
& CL & 0 & $\mathbf{4}$ & 0 & 0 & 0 & 0 & 0 & 0 & 0 & 0 & $\mathbf{1 0 0 . 0}$ \\
& CR & 0 & 0 & $\mathbf{4}$ & 0 & 0 & 0 & 0 & 0 & 0 & 0 & $\mathbf{1 0 0 . 0}$ \\
& WG & 0 & 0 & 0 & $\mathbf{4}$ & 0 & 0 & 0 & 0 & 0 & 0 & $\mathbf{1 0 0 . 0}$ \\
& PX & 0 & 0 & 0 & 0 & $\mathbf{4}$ & 0 & 0 & 0 & 0 & 0 & $\mathbf{1 0 0 . 0}$ \\
& PN & 0 & 0 & 0 & 0 & 0 & $\mathbf{3}$ & 0 & 0 & 0 & 1 & $\mathbf{7 5 . 0}$ \\
& TE & 0 & 0 & 0 & 0 & 0 & 1 & $\mathbf{3}$ & 0 & 0 & 0 & $\mathbf{7 5 . 0}$ \\
& TR & 0 & 0 & 0 & 0 & 0 & 0 & 0 & $\mathbf{4}$ & 0 & 0 & $\mathbf{1 0 0 . 0}$ \\
& VO & 0 & 0 & 0 & 1 & 0 & 0 & 0 & 0 & $\mathbf{3}$ & 0 & $\mathbf{7 5 . 0}$ \\
& VU & 0 & 0 & 0 & 0 & 0 & 0 & 0 & 0 & 0 & $\mathbf{4}$ & $\mathbf{1 0 0 . 0}$ \\
\hline
\end{tabular}

CS: Cabernet Sauvignon; CL: Caladoc; CR: Carmenere; WG: White Grenache; PX: Pedro Ximenez; PN: Pinot Noir; TE: Tempranillo; TR: Treixadura; VO: Viognier; VU: Viura.

The detailed accuracy per class for the external validation is displayed in Table 4 . Confirming the results in the confusion matrix of Table 3, seven varieties obtained a value of one in the true positive rate, but two of them-White Grenache and Viura-did not achieve a perfect score, because some samples were misclassified as those varieties. It is remarkable that two classes that obtained a precision value of one (Tempranillo and Viognier) did not reach a full true positive rate, meaning that all samples that were classified as Tempranillo and Viognier were in effect leaves of those varieties. According to the AUC values, eight out of 10 classes yielded the perfect score, increasing the average AUC for all of the classes to the 0.997 mark.

Table 4. Detailed accuracy by class of the grapevine varietal classification using support vector machines and an external validation of 40 samples $(n=40)$.

\begin{tabular}{lcccc}
\hline Class & True Positive Rate & False Positive rate & Precision & AUC \\
\hline Cabernet Sauvignon (CS) & 1.000 & 0.000 & 1.000 & 1.000 \\
Caladoc (CL) & 1.000 & 0.000 & 1.000 & 1.000 \\
Carmenere (CR) & 1.000 & 0.000 & 1.000 & 1.000 \\
White Grenache (WG) & 1.000 & 0.028 & 0.800 & 0.993 \\
Pedro Ximenez (PX) & 1.000 & 0.000 & 1.000 & 1.000 \\
Pinot Noir (PN) & 0.750 & 0.028 & 0.750 & 0.972 \\
Tempranillo (TE) & 0.750 & 0.000 & 1.000 & 1.000 \\
Treixadura (TR) & 1.000 & 0.000 & 1.000 & 1.000 \\
Viognier (VO) & 0.750 & 0.000 & 1.000 & 1.000 \\
Viura (VU) & 1.000 & 0.028 & 0.800 & 1.000 \\
\hline Weighted average & 0.925 & 0.008 & 0.935 & 0.997 \\
\hline
\end{tabular}

AUC: area under the receiver operating characteristic (ROC) curve.

\subsection{Assessment of Grapevine Water Status}

\subsubsection{Multi-Variety Model}

The spectral outlier analysis tagged one sample of Godello and another of Grenache as mismeasured spectra, so both were removed before the development of the regression model. The ranges of $\psi_{\text {stem }}$ per variety are shown in Table 5. It can be observed that Cermenere and Tempranillo were the most water stressed varieties, while Pedro Ximenez and Godello experienced no water scarcity. Grenache and Marselan exhibited a similar $\psi_{\text {stem }}$ range, indicative of an incipient moderate water stem. 
Table 5. Stem water potential $\left(\psi_{\text {stem }}\right)$ ranges per variety.

\begin{tabular}{ccccccc}
\hline \multirow{2}{*}{$\psi_{\text {stem }}$} & \multicolumn{5}{c}{ Variety } \\
\cline { 2 - 7 } & Godello & Pedro Ximenez & Grenache & Carmenere & Tempranillo & Marselan \\
\hline Min & -0.90 & -0.65 & -1.15 & -1.45 & -1.85 & -1.02 \\
\hline Max & -0.62 & -0.42 & -0.85 & -1.10 & -1.62 & -0.85 \\
\hline
\end{tabular}

Table 6 shows the statistical summary for the $\psi_{\text {stem }}$ values of the sampled population and the result of the calibration, cross- and external validations of the stem water potential estimation using a rotation forest and M5 trees.

Table 6. Statistic overview and results of the $\psi_{\text {stem }}(\mathrm{MPa})$ estimation using a rotation forest and M5 trees.

\begin{tabular}{|c|c|c|c|c|c|c|c|c|c|c|}
\hline & & & & & \multicolumn{6}{|c|}{ Rotation Forest and M5 Trees } \\
\hline \multicolumn{5}{|c|}{ Statistics } & \multicolumn{2}{|c|}{$\begin{array}{l}\text { Calibration } \\
(\mathrm{n}=94)\end{array}$} & \multicolumn{2}{|c|}{$\begin{array}{c}\text { 5-Fold } \\
\text { Cross-Validation } \\
(\mathrm{n}=94)\end{array}$} & \multicolumn{2}{|c|}{$\begin{array}{c}\text { External } \\
\text { Validation } \\
(\mathrm{n}=24)\end{array}$} \\
\hline $\mathbf{n}$ & Min & Max & Mean & SD & $\mathbf{R}^{2}$ & RMSE & $\mathbf{R}^{2}$ & RMSE & $\mathbf{R}^{2}$ & RMSE \\
\hline 118 & -1.85 & -0.42 & -1.03 & 0.396 & 0.97 & 0.083 & 0.84 & 0.164 & 0.84 & 0.165 \\
\hline
\end{tabular}

n: number of samples; Min: minimum; Max: maximum; SD: standard deviation; RMSE: root-mean-square error in MPa.

The determination coefficient $\left(R^{2}\right)$ and root-mean-square error (RMSE) of calibration were 0.97 and 0.083 , respectively. For both validation processes, these values were $R^{2}=0.84$, RMSE: 0.164 , for the cross-validation (having a training dataset with 94 samples) and $R^{2}=0.84$, RMSE: 0.165 , for external validation (with a test dataset having 24 samples).

The regression plots for cross- and external validation models are displayed in Figure 2, along with the prediction bands at a $95 \%$ of confidence. To perform a deeper analysis of the predicted outcomes, a manual clustering into four groups was performed for the samples according to the absolute error value $|\varepsilon|=$ measured $\psi_{\text {stem }}$ - predicted $\psi_{\text {stem }}$ : minimal error $(|\varepsilon|<0.1)$. low error $(0.1 \leq|\varepsilon|<0.2)$, moderate error $(0.2 \leq|\varepsilon|<0.4)$ and high error $(|\varepsilon| \geq 0.4)$. For the cross-validation (Figure 2a), 40 samples obtained a minimal error (42.6\% of the samples), 34 a low error $(36.2 \%), 18$ a moderate error $(19.1 \%)$ and 2 a high error (2.1\%). In the external validation (Figure $2 \mathrm{~b}$ ), 16 samples obtained a minimal error (66.7\%), 5 a low error (20.8\%), 2 a moderate error $(8.3 \%)$ and 1 a high error (4.2\%). Two samples for the cross-validation (Figure $2 a$ ) and another one for the external validation (Figure 2a) were present in the regression models, samples that obtained an absolute error value greater than $0.4 \mathrm{MPa}$. In both plots, these high error samples were driven by the divergence of the regression line from the 1:1 line. Further, the samples with the minimal and low error values did considerably fit better to the diagonal line 1:1 than the high error samples. The prediction bands at a $95 \%$ confidence only excluded a few samples in both cases. For the cross-validation (Figure 2a), four samples lied out of the precision bands, meaning that $95.7 \%$ of the samples were inside both bands. For the external validation results (Figure $2 \mathrm{~b}$ ), $95.8 \%$ of the samples lied between the $95 \%$ confidence bands, while only one sample was kept out. 

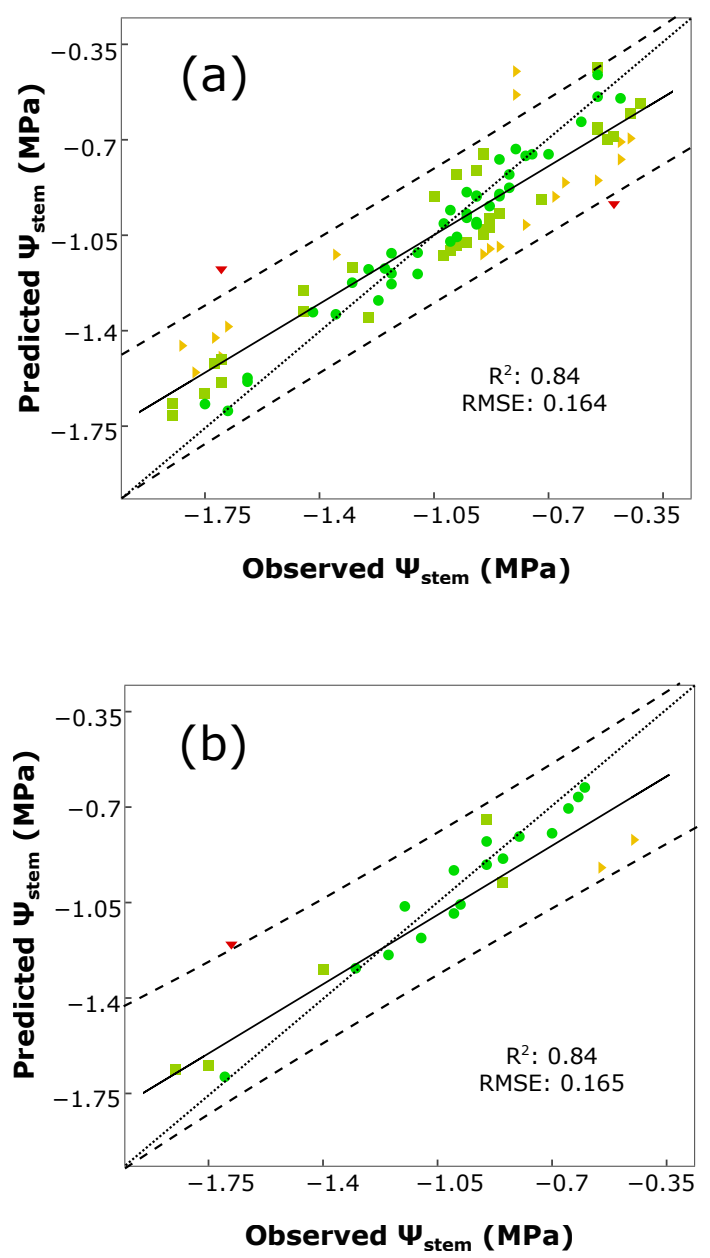

Figure 2. Regression plot for $\psi_{\text {stem }}$ estimation using a Rotation Forest and M5 trees with a 5-fold cross (a) and external (b) validations. Prediction confidence bands are shown at a 95\% level (dashed lines). Solid line represents the regression line and dotted line refers to the 1:1 line. Each points' color and shape refers to its absolute error value $|\varepsilon|$ (the absolute value of the difference between the actual value and the predicted one) in MPa: green $\bullet:|\varepsilon|<0.1$, minimal error; olive $\mathbf{\square}: 0.1 \leq|\varepsilon|<0.2$, low error; orange $\mathbf{~ : ~} 0.2 \leq|\varepsilon|<0.4$, moderate error; red $\mathbf{\nabla}:|\varepsilon| \geq 0.4$, high error.

\subsubsection{Variety-Specific Multi-Vineyard Model}

Table 7 shows the statistical summary for the $\psi_{\text {stem }}$ values of the sampled population from the variety-specific model (involving samples of Tempranillo taken both in 2012 and 2015 from two different vineyards) and the result of the calibration, cross- and external validations of the stem water potential estimation using a rotation forest and M5 trees.

Table 7. Statistic overview and results of the $\psi_{\text {stem }}(\mathrm{MPa})$ estimation for the variety-specific model (Tempranillo) using a rotation forest and M5 trees.

\begin{tabular}{|c|c|c|c|c|c|c|c|c|c|c|}
\hline \multirow{2}{*}{\multicolumn{5}{|c|}{ Statistics }} & \multicolumn{6}{|c|}{ Rotation Forest and M5 Trees } \\
\hline & & & & & \multicolumn{2}{|c|}{$\begin{array}{l}\text { Calibration } \\
(n=45)\end{array}$} & \multicolumn{2}{|c|}{$\begin{array}{c}\text { 5-Fold } \\
\text { Cross-Validation } \\
(\mathrm{n}=45)\end{array}$} & \multicolumn{2}{|c|}{$\begin{array}{l}\text { External } \\
\text { Validation } \\
(\mathrm{n}=11)\end{array}$} \\
\hline $\mathbf{n}$ & Min & Max & Mean & SD & $\mathbf{R}^{2}$ & RMSE & $\mathbf{R}^{2}$ & RMSE & $\mathbf{R}^{2}$ & RMSE \\
\hline 56 & -1.85 & -0.8 & -1.447 & 0.314 & 0.92 & 0.098 & 0.76 & 0.159 & 0.79 & 0.168 \\
\hline
\end{tabular}

n: number of samples; Min: minimum; Max: maximum; SD: standard deviation; RMSE: root-mean-square error in MPa. 
The range of $\psi_{\text {stem }}$, in Table 7 , illustrates a population of grapevines involving plants of very different water status, from no stressed plants $\left(\psi_{\text {stem }}=-0.8 \mathrm{MPa}\right)$ to severely stressed plants $\left(\psi_{\text {stem }}=-1.85 \mathrm{MPa}\right)$, the mean being $\psi_{\text {stem }}(-1.45 \mathrm{MPa})$, indicative of high water stress.

The determination coefficient $\mathrm{R}^{2}$ and RMSE of calibration were 0.92 and 0.098 , respectively, while for both validation processes, these values were $\mathrm{R}^{2}=0.76$, RMSE: 0.159 (cross-validation), and $\mathrm{R}^{2}=0.79$, RMSE: 0.168 (external validation).
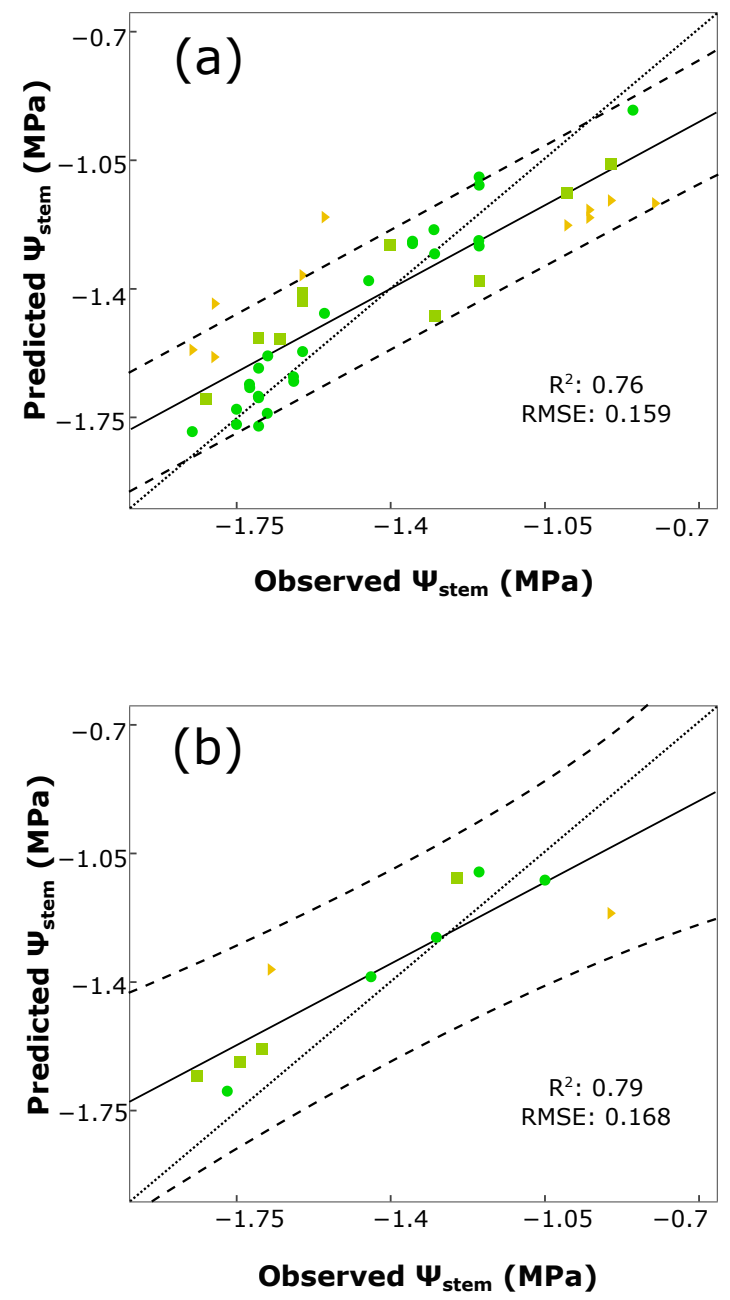

Figure 3. Regression plot for $\psi_{\text {stem }}$ estimation of the variety-specific model (Tempranillo) using a Rotation Forest and M5 trees with a 5-fold cross (a) and external (b) validations. Prediction confidence bands are shown at a 95\% level (dashed lines). Solid line represents the regression line and dotted line refers to the 1:1 line. Each points' color and shape refers to its absolute error value $|\varepsilon|$ (the absolute value of the difference between the actual value and the predicted one) in MPa: green $\bullet:|\varepsilon|<0.1$, minimal error; olive $\mathbf{\square}$ : $0.1 \leq|\varepsilon|<0.2$, low error; orange $\mathbf{\nabla}: 0.2 \leq|\varepsilon|<0.4$, moderate error; red $\mathbf{\nabla}$ : $|\varepsilon| \geq 0.4$, high error.

Figure 3 shows the regression plots for the two validation processes and their prediction bands at a 95\% of confidence. In the 5-fold cross-validation (Figure 3a), 25 samples obtained a minimal error value, 10 a low error and 10 a moderate one. Five out of 45 samples lied out the prediction bands, keeping $88.9 \%$ of the samples inside them. In Figure $3 b$ (external validation), all of the samples were inside the confidence bands, where 5 of them obtained a minimal error value, 4 a low one and 2 a moderate error value. 


\section{Discussion}

In this work, the appraisal of two important phenotyping features in agriculture-grapevine varietal discrimination and water status assessment-has been aimed at from an innovative approach that successfully combines an in-field measurement, using a proximal and non-invasive sensor, with different data mining processing methods. The results obtained have displayed the potential of effectively applying data mining techniques upon the spectral information retrieved from a non-destructive and proximal NIR sensor for grapevine plant phenotyping of two key traits.

Regarding variety classification, most of the widely-used methods for grapevine varietal discrimination have traditionally been either destructive or time-consuming, like classic ampelometry [40] (which is subjected to expert visual description, but still prone to a considerable level of bias due to its human nature), DNA analysis [41] or wet chemistry techniques [42] (carried out by trained people and through destructive methods).

In our work, the 10-class variety classification models using SVMs from non-invasively acquired leaf spectra have yielded $88.7 \%$ and 92.5 values of correctly discriminated samples for cross- and external validations, respectively. These high percentages allow one to be reasonably optimistic about the suitability of SVMs for the grapevine varietal classification. These correctly-classified percentages are also supported by the high scores of additional classification statistics, such as the average precision (obtaining in several cases a perfect score and high mean values) and AUCs (an average of 0.991 and 0.997 for cross- and external validation, respectively).

Only very recently, grapevine varietal classification has been attempted by hyperspectral imaging [24] and an NIR portable spectrophotometer [43]. In [24], hyperspectral imaging in the range between $280 \mathrm{~nm}$ and $1028 \mathrm{~nm}$ was used along with PLS for the classification of 300 leaves from three different varieties (Tempranillo, Grenache and Cabernet Sauvignon), under laboratory conditions. The cross-validation method used (Monte Carlo) yielded more than $92 \%$ of correctly classified samples in all cases. The outcomes reached in the present work, even when a large number of varieties was selected for the training, highlights the accuracy shown by data mining techniques for the same goal, particularly when the spectra were collected in the field and in a non-destructive way, different from [24], where a hyperspectral camera was used indoors under controlled illumination conditions. In [43], the authors used a portable NIR spectrophotometer of the same range as the one in this work for the acquisition of leaves' spectra. Artificial neural networks (ANNs) and sequential minimal optimization for the training of SVMs were tested as classification algorithms for the development of two grapevine discrimination models for two different approaches: a site-specific model for 20 varieties (yielding $87.25 \%$ of correctly classified samples, using ANNs) and a global model using six varieties from different vineyards and seasons (obtaining 77.08\%, again with ANNs). The higher percentages obtained in the present study could be explained by the selected SVM algorithm, v-SVM algorithm, versus sequential minimal optimization, as well as the reduced number of classes.

Varietal discrimination using NIR spectroscopy has also been recently performed for waxy corn seed [44] using SVM and in strawberry [45] and plum [46] using PLS-D. From these works, it is remarkable that a purer data mining technique, SVMs [44], behaved better than the statistical method PLS, commonly used in spectroscopy and chemometrics, confirming the high suitability and adaptability of machine learning approaches for any kind of problem and specifically NIR spectroscopy. Five- and four-class varietal discrimination using PLS-DA was achieved in $[45,46]$ obtaining $69 \%$ and up to $96.5 \%$ values of correctly classified samples, respectively, presenting lower than and similar accuracies as in the present grapevine varietal discrimination, but taking into account that the number of varieties was reduced by half.

The proven flexibility, generalization capability and accuracy in so many dissimilar fields for discrimination issues given by data mining techniques, and confirmed by the results of the grapevine varietal classification via SVMs, demonstrates how well the numerous data mining algorithms fit in classification problems, specifically when working with NIR spectroscopy from proximal sensors. 
Current water status assessment methods are mostly destructive, labor intensive, thus expensive, and, in many cases, only capable of being implemented in a limited number of samples, jeopardizing their representativeness and not suitable for characterizing the spatial variability of a vineyard's water status. Therefore, new non-invasive and fast approaches are needed.

For the regression of $\psi_{\text {stem }}$ conducted through rotation forests and M5 trees, the calibration $\mathrm{R}^{2}$ and RMSE reached the 0.97 and 0.083 values, respectively, while both validation results were virtually identical $\left(\mathrm{R}^{2}=0.84\right.$; RMSE $\left.=0.165\right)$. A relatively large divergence between calibration and validation results was found, where the latter's RMSE nearly doubled that of the calibration. Still, this difference of $0.082 \mathrm{MPa}$, although, as said, being relatively wide, remains small in absolute terms, particularly when compared to the standard deviation of the population $\psi_{\text {stem }}$ values $(0.396)$, that is almost five-times larger. The high score of the determination coefficient of calibration is an aspect that could be generally expected when using data mining and machine learning techniques. Moreover, the training of decision trees is very sensitive to the examples used as input, having a high importance for the algorithm (that tries to extract underlying rules and correlations between the independent and dependent variables), so high results are likely to be obtained when testing with the same set that the algorithm was trained. The use of the calibration results should be carefully treated when applying data mining algorithms, and they should be contrasted with values that came from validation processes. However, the high results obtained for both cross- and external validations concede a considerable level of confidence in the suppression of any overfitting problem.

Additionally, the good outcomes obtained from the variety-specific model (although slightly lower than the multi-varietal one) show the robustness of the application of data mining algorithms for the accurate prediction of $\psi_{\text {stem }}$ of samples from different seasons and locations when properly training the models with both kinds of examples. This could enable affirming that support vector machines are able to assess the grapevine water status within one variety and to discard the variety as a driving factor in good water status prediction. Still, should the variety-specific model have a higher number of samples and/or a wider range in the water status reference parameter $\left(\psi_{\text {stem }}\right)$, the model's performance would have probably yielded higher $\mathrm{R}^{2}$ and RMSE values. It should not be omitted that the Tempranillo dataset, compared to the multi-varietal one, contained a lower number of samples (56 vs. 120$)$ and a narrower $\psi_{\text {stem }}$ range $([-1.85,-0.8]$ vs. $[-1.85,-0.42], \mathrm{MPa})$.

Stem water potential, as an indicator of plant water stress, has been previously predicted by NIR-based models developed using PLS regression [26-29], returning determination coefficients between 0.71 and 0.85 (and error values around 0.1 and $0.2 \mathrm{MPa}$ ). The in-field multi-varietal study performed in the current work, using rotation forests and M5 trees, returned a similar determination coefficient for cross- and external validations, highlighting that a considerably higher number of varieties was used. The fact that both studies ([26] and this one) clearly resulted in high $\psi_{\text {stem }}$ correlations from two different and scarcely overlapped NIR regions may drive one to conclude the adequate suitability of NIR spectral measurements from non-destructive sensors in water status prediction.

Models for the grapevine $\psi_{\text {leaf }}$ [28] and $\psi_{\text {stem }}$ in olive trees [27] were developed by VIS/NIR spectroscopy. These works have in common the use of PLS as a model training method, returning moderated values of cross-validation correlation ( $\mathrm{R}^{2}$ from 0.45 to 0.74$)$ that are noticeably surpassed by the results from the rotation forest and M5 trees models described in this work, allowing one to confirm the effective application of data mining techniques to NIR spectral data for the estimation of $\psi_{\text {stem }}$, hence the assessment of plant water status. Additionally, it must be highlighted that the spectral range used in this study $(1600$ to $2400 \mathrm{~nm}$ ) completely covered the absorption band (O-H) corresponding to the water vibrational band $(1940 \mathrm{~nm})$ [47], which could be one of the reasons for the high sensitivity in $\psi_{\text {stem }}$ changes; thus, a good predictive model could be obtained from this spectral range.

To the best of our knowledge, scarce studies have made use of data mining algorithms for water status assessment. In [48], the authors built an artificial neural network for the in-lab relative water 
content (RWC) estimation from grapevine leaf's hyperspectral imaging working in the range from $900 \mathrm{~nm}$ to $1700 \mathrm{~nm}$. The authors asserted that the generated models (with average absolute error below the $3 \%$ mark) were shown to be leaf side, varietal and even clone dependent. Although no direct comparison can be made with the present work, because RWC was used as a water status indicator instead of $\psi_{\text {stem }}$, both results have displayed the accuracy of the combination of NIR spectroscopy along with data mining and machine learning techniques for the reliable assessment of plant, grapevine specifically, water status.

The selection of a proper estimation method for a concrete algorithm and dataset is crucial for the evaluation of the results. In multivariate chemometrics, a classic approach of performance evaluation has been the dataset split into calibration (or training) and test partitions [49]. Although the use of the same dataset for the training and testing is not generally recommended, because the obtained results are overly optimistic [9], its value could be considered as an upper limit to what may be expected in other settings (e.g., cross- and external validation). $k$-fold and leave-one-out cross-validation methods [50] have been broadly extended in data mining and chemometrics. The selection of $k=5$ for the cross-validation in the present work, maintaining the 80:20 ratio as in the external validation, can lead to a higher consistency and reliability on the obtained results in both experiments.

It is also remarkable the duality brought by the spectral measurements obtained with the same NIR sensor. The capability of effectively addressing these two grapevine phenotyping traits from a single leaf spectral measurement along with its rapid, non-destructive and in-field nature makes the almost direct implementation of a grapevine phenotyping system on an NIR device a reasonable goal supported by the precision obtained in the developed models and the characterization of concrete and sufficient sets of samples for the training.

\section{Conclusions}

Combining non-invasive sensors and data mining algorithms may be a powerful tool that could allow one to perform grapevine phenotyping tasks and forward the results to the user directly in the field. The proven good behavior of different data mining techniques along with the non-invasive, fast and responsive nature of a portable NIR sensor for the in-field grapevine varietal classification and water status assessment opens a way for the direct application of the models in embedded portable systems.

The two phenotyping traits addressed in this study-grapevine discrimination and water status assessment-deserve major attention in modern viticulture, as they are key factors in breeding, grape quality production and sustainability. Their robust prediction from non-invasively acquired data is expected to have a positive impact in precision viticulture and, extensively, several other agricultural areas. These are very likely to benefit from the application of the results obtained by the prediction models due to their almost direct application of the training process via data mining to a portable NIR device.

Acknowledgments: This work has received funding from the Spanish Ministry of Economy and Competitiveness (MINECO) under the INNGRAPEproject (RTC-2014-3058-2). The authors want to thank Vitis Navarra S.L. (Larraga, Navarra, Spain) for providing the vineyards to carry out this study. Special thanks to Victor Sicilia, Teresa Díaz and Borja Millan for their help in the vineyard during data acquisition.

Author Contributions: Maria P. Diago and Javier Tardaguila conceived of and designed the experiments. Maria P. Diago performed the experiments. Salvador Gutiérrez and Juan Fernández-Novales analyzed the data. Javier Tardaguila contributed reagents/materials/analysis tools. Salvador Gutiérrez, Javier Tardaguila, Juan Fernández-Novales and Maria P. Diago wrote the paper.

Conflicts of Interest: The authors declare no conflict of interest.

\section{References}

1. Walter, A.; Liebisch, F.; Hund, A. Plant phenotyping: From bean weighing to image analysis. Plant Methods 2015, 11, doi:10.1186/s13007-015-0056-8. 
2. Minervini, M.; Scharr, H.; Tsaftaris, S. Image Analysis: The New Bottleneck in Plant Phenotyping [Applications Corner]. IEEE Signal Process. Mag. 2015, 32, 126-131.

3. Fiorani, F.; Schurr, U. Future scenarios for plant phenotyping. Annu. Rev. Plant Biol. 2013, 64, $267-291$.

4. Roberts, C.A.; Workman, J., Jr.; Reeves, J.B., III. Near-Infrared Spectroscopy in Agriculture; American Society of Agronomy: Madison, WI, USA, 2004.

5. Cozzolino, D.; Esler, M.; Dambergs, R.; Cynkar, W.; Boehm, D.; Francis, I.; Gishen, M. Prediction of colour and $\mathrm{pH}$ in grapes using a diode array spectrophotometer $(400-1100 \mathrm{~nm})$. J. Near Infrared Spectrosc. 2004, 12, 105-112.

6. Smith, J.; Schmidtke, L.; Müller, M.; Holzapfel, B. Measurement of the concentration of nutrients in grapevine petioles by attenuated total reflectance Fourier transform infrared spectroscopy and chemometrics. Aust. J. Grape Wine Res. 2014, 20, 299-309.

7. Rustioni, L.; Rocchi, L.; Guffanti, E.; Cola, G.; Failla, O. Characterization of grape (Vitis vinifera L.) berry sunburn symptoms by reflectance. J. Agric. Food Chem. 2014, 62, 3043-3046.

8. Han, J.; Kamber, M.; Pei, J. Data Mining: Concepts and Techniques: Concepts and Techniques; Elsevier: Harrisburg, PA, USA, 2011.

9. Witten, I.H.; Frank, E. Data Mining: Practical Machine Learning Tools and Techniques; Morgan Kaufmann: Burlington, VT, USA, 2005.

10. Quinlan, J.R. Induction of decision trees. Mach. Learn. 1986, 1, 81-106.

11. Rokach, L. Decision forest: Twenty years of research. Inf. Fusion 2016, 27, 111-125.

12. Rumelhart, D.E.; Hinton, G.E.; Williams, R.J. Learning representations by back-propagating errors. Nature 1986, doi:10.1038/323533a0.

13. Cortes, C.; Vapnik, V. Support-vector networks. Mach. Learn. 1995, 20, 273-297.

14. Lavrač, N. Selected techniques for data mining in medicine. Artif. Intell. Med. 1999, 16, 3-23.

15. Giudici, P. Applied Data Mining: Statistical Methods for Business and Industry; John Wiley \& Sons: New York, NY, USA, 2005.

16. Hirschman, L.; Park, J.C.; Tsujii, J.; Wong, L.; Wu, C.H. Accomplishments and challenges in literature data mining for biology. Bioinformatics 2002, 18, 1553-1561.

17. Boser, B.E.; Guyon, I.M.; Vapnik, V.N. A training algorithm for optimal margin classifiers. In Proceedings of the Fifth Annual Workshop on Computational Learning Theory. Pittsburgh, PA, USA, 27-29 July 1992; pp. 144-152.

18. Rodriguez, J.J.; Kuncheva, L.I.; Alonso, C.J. Rotation forest: A new classifier ensemble method. IEEE Trans. Pattern Anal. Mach. Intell. 2006, 28, 1619-1630.

19. Quinlan, J.R. Learning with continuous classes. In Proceedings of the 5th Australian Joint Conference on Artificial Intelligence, Hobart, Australia, 16-18 November 1992; pp. 343-348.

20. Wold, S.; Sjöström, M.; Eriksson, L. PLS-regression: A basic tool of chemometrics. Chemom. Intell. Lab. Syst. 2001, 58, 109-130.

21. Kisi, O. Pan evaporation modeling using least square support vector machine, multivariate adaptive regression splines and M5 model tree. J. Hydrol. 2015, 528, 312-320.

22. Štravs, L.; Brilly, M. Development of a low-flow forecasting model using the M5 machine learning method. Hydrol. Sci. J. 2007, 52, 466-477.

23. Bhattacharya, B.; Solomatine, D.P. Neural networks and M5 model trees in modeling water level-discharge relationship. Neurocomputing 2005, 63, 381-396.

24. Diago, M.; Fernandes, A.; Millan, B.; Tardaguila, J.; Melo-Pinto, P. Identification of grapevine varieties using leaf spectroscopy and partial least squares. Comput. Electron. Agric. 2013, 99, 7-13.

25. Jones, H.G. Irrigation scheduling: Advantages and pitfalls of plant-based methods. J. Exp. Bot. 2004, 55, $2427-2436$.

26. De Bei, R.; Cozzolino, D.; Sullivan, W.; Cynkar, W.; Fuentes, S.; Dambergs, R.; Pech, J.; Tyerman, S. Non-destructive measurement of grapevine water potential using near infrared spectroscopy. Aust. J. Grape Wine Res. 2011, 17, 62-71.

27. Poblete-Echeverría, C.; Ortega-Farías, S.; Lobos, G.; Romero, S.; Ahumada, L.; Escobar, A.; Fuentes, S. Non-invasive method to monitor plant water potential of an olive orchard using visible and near infrared spectroscopy analysis. Acta Hortic. 2014, 1057, 363-368.

28. Vila, H.; Hugalde, I.; di Filippo, M. Estimation of leaf water potential by thermographic and spectral measurements in grapevine. RIA Rev. de Investig. Agropecu. 2011, 37, 46-53. 
29. Santos, A.O.; Kaye, O. Grapevine leaf water potential based upon near infrared spectroscopy. Sci. Agric. 2009, 66, 287-292.

30. Perez, D.; Sanchez, M.; Cano, G.; Garrido, A. Authentication of Green Asparagus Varieties by Near-Infrared Reflectance Spectroscopy. J. Food Sci. 2001, 66, 323-327.

31. Naes, T.; Isaksson, T.; Fearn, T.; Davies, T. A User Friendly Guide to Multivariate Calibration and Classification; NIR publications: Chichester, UK, 2002.

32. Scholander, P.F.; Bradstreet, E.D.; Hemmingsen, E.; Hammel, H. Sap pressure in vascular plants negative hydrostatic pressure can be measured in plants. Science 1965, 148, 339-346.

33. Rinnan, Å.; van den Berg, F.; Engelsen, S.B. Review of the most common pre-processing techniques for near-infrared spectra. TrAC Trends Anal. Chem. 2009, 28, 1201-1222.

34. Barnes, R.; Dhanoa, M.; Lister, S. Standard Normal Variate Transformation and De-trending of Near-Infrared Diffuse Reflectance Spectra. Appl. Spectrosc. 1989, 43, 772-777.

35. Dhanoa, M.; Lister, S.; Barnes, R. On the scales associated with near-infrared reflectance difference spectra. Appl. Spectrosc. 1995, 49, 765-772.

36. Savitzky, A.; Golay, M. Smoothing and differentiation of data by simplified least squares procedures. Anal. Chem. 1964, 36, 1627-1639.

37. Williamson, R.; Bartlett, P. New support vector algorithms. Neural Comput. 2000, 12, 1207-1245.

38. Chang, C.C.; Lin, C.J. LIBSVM: A library for support vector machines. ACM Trans. Intell. Syst. Technol. 2011, 2, doi:10.1145/1961189.1961199.

39. Hall, M.; Frank, E.; Holmes, G.; Pfahringer, B.; Reutemann, P.; Witten, I.H. The WEKA data mining software: An update. ACM SIGKDD Explor. Newsl. 2009, 11, 10-18.

40. Galet, P. A practical Ampelography; Cornell University Press: New York, NY, USA, 1979.

41. Pelsy, F.; Hocquigny, S.; Moncada, X.; Barbeau, G.; Forget, D.; Hinrichsen, P.; Merdinoglu, D. An extensive study of the genetic diversity within seven French wine grape variety collections. Theor. Appl. Genet. 2010, $120,1219-1231$.

42. Altube, H.; Cabello, F.; Ortiz, J. Caracterización de variedades y portainjertos de vid mediante isoenzimas de los sarmientos. Vitis 1991, 30, 203-212.

43. Gutiérrez, S.; Tardaguila, J.; Fernández-Novales, J.; Diago, M.P. Support vector machine and artificial neural network models for the classification of grapevine varieties using a portable NIR spectrophotometer. PLOS ONE 2015, 10, doi: 10.1371/journal.pone.0143197.

44. Yang, X.; Hong, H.; You, Z.; Cheng, F. Spectral and Image Integrated Analysis of Hyperspectral Data for Waxy Corn Seed Variety Classification. Sensors 2015, 15, 15578-15594.

45. Sánchez, M.T.; de la Haba, M.J.; Benítez-López, M.; Fernández-Novales, J.; Garrido-Varo, A.; Pérez-Marín, D. Non-destructive characterization and quality control of intact strawberries based on NIR spectral data. J. Food Eng. 2012, 110, 102-108.

46. Pérez-Marín, D.; Paz, P.; Guerrero, J.E.; Garrido-Varo, A.; Sánchez, M.T. Miniature handheld NIR sensor for the on-site non-destructive assessment of post-harvest quality and refrigerated storage behavior in plums. J. Food Eng. 2010, 99, 294-302.

47. Shenk, J.S.; Workman, J.J.; Westerhaus, M.O. Application of NIR spectroscopy to agricultural products. Pract. Spectrosc. Ser. 2001, 27, 419-474.

48. Diago, M.; Pou, A.; Millan, B.; Tardaguila, J.; Fernandes, A.; Melo-Pinto, P. Assessment of grapevine water status from hyperspectral imaging of leaves. Acta Hortic. 2014, 1038, 89-96.

49. Brereton, R.G. Applied Chemometrics for Scientists; John Wiley \& Sons: New York, NY, USA, 2007.

50. Wong, T.T. Performance evaluation of classification algorithms by $k$-fold and leave-one-out cross validation. Pattern Recognit. 2015, 48, 2839-2846.

(C) 2016 by the authors; licensee MDPI, Basel, Switzerland. This article is an open access article distributed under the terms and conditions of the Creative Commons by Attribution (CC-BY) license (http://creativecommons.org/licenses/by/4.0/). 